\title{
Audio Content Curation in Digital Music Streaming Applications Music Recommendations in Spotify Playlists
}

\author{
Reno Dalu Maharso ${ }^{1, *}$ Irwansyah $^{2}$
}

\author{
${ }^{1}$ Universitas Indonesia \\ ${ }^{2}$ Universitas Indonesia \\ *Corresponding author. Email: reno.dalu@gmail.com
}

\begin{abstract}
Digital audio streaming services grant digital media users access to seemingly infinite music if they are connected to the Internet. But abundant music brings a problem: users facing too many songs from which to choose. This problem is solved by curating music to suit users, thus introducing a recommendation system in the form of playlists to help users navigate in consuming relevant music to them. This conceptual article highlights the role of music curation as an important aspect of digital music streaming, represented in the form of playlists. A literature review is the research method as the article revisits such concepts as streaming, digital music streaming, playlists, and Bhaskar's idea of curation. The article also introduces the Wesley-Maclean concept of gatekeeping digital music streaming's content curation. The result demonstrates that curation is a mixture of using algorithms that see music consumption as statistics and the human touch to curate music qualitatively. Curation function can also be developed as a kind of service with significant commercial value. This article aims to enrich the perspective of digital content curation that contributes to research on digital content in general.
\end{abstract}

Keywords: Curation, Playlist, Gatekeeping, Music, Streaming Introduction

\section{INTRODUCTION}

Digital music streaming applications allow digital media users to access a seemingly unlimited amount of music. If constantly connected to the Internet, users can get up-to-date music content continuously. However, an abundant amount of audio content may lead users to face the dilemma of having too many songs from which to choose (Herbert, Lotz, \& Marshall 2018). This problem is solved by content recommendation systems that use playlists to guide users in consuming music. The playlist system exemplifies content curation function in digital media, recommending music content in digital streaming applications. The playlist system and recommendations can be seen as part of a changing consumption pattern that endorses a curation system to help users navigate certain content. A recommendation system marked by curated catalogs is a new perspective that has become characteristic of streaming services (Lobato 2018).

This article shows that content curation occurs in digital streaming media as a practice of selecting, organizing, and adding value to large content through aggregated lists
(Bhaskar, 2016). In the digital audio streaming application, selected music contents are put together into playlists. This article also emphasizes that the best practice for curating music content in a digital music streaming application is by combining curators' work with an algorithm's work. This is supported by books and news sources that mention the importance of the human aspect in building music recommendations for users of digital music streaming services (Buzzfeed News 2017; Eriksson et al. 2019; Ugwu 2016). Streaming music applications include Apple Music, Pandora, Soundcloud, and Tidal (Hall 2019), but this article focuses on Spotify because its literature review materials, as secondary data, are more varied and offer greater quantity and quality than other digital music streaming applications.

\subsection{Digital Music Streaming}

Digital music is currently very popular because it offers unlimited access to nearly unlimited music content (Herbert et al. 2018), and it can be accessed anytime a user is connected to the Internet. Consuming digital music streaming is an activity that accesses content from a source 
(Beer \& Burrows 2013). Meanwhile, young adults' listening to music emphasizes variability of options, oriented to individuals' networks and their taste preferences (Werner \& Johansson 2016).

With a population of 268 million in 2019, Indonesia is certainly a potential market for digital music streaming applications because approximately 150 million (55\%) Indonesians use the Internet. As many as $60 \%$ of adults use smartphones, and $22 \%$ have a laptop or personal computer. Moreover, Indonesian Internet users can listen to streaming applications for 1 hour, 22 minutes a day (Kemp 2019). This data is consistent with Spotify Indonesia's claim that, on average, Indonesian consumers listen to the application for 90 minutes daily (Bohang 2016), thus indicating a potential market that companies providing digital music streaming services want to enter. One example is Indihome, which tried to enter the market with its streaming application called Melon ID (Indihome n.d.).

Consuming music through streaming applications has eliminated reasons for users to own physical artifacts such as cassettes, compact discs (CDs), and even music files in computers or electronic devices (Léveillé Gauvin 2018). Consistent with consumption changes, digital media users are no longer "music owners" but "music users." Moreover, "consuming music" means users need to purchase access from streaming services providers (Arditi 2017).

Adopting streaming technology also means transforming users' music listening experience. Introduction of algorithms and recommendation systems in streaming systems has changed how users choose music (Torres 2018). Content curation systems navigate users through a series of recommendations and create playlists (Herbert et al. 2018). Digital music streaming services develop a content curation system in their platforms to cope with abundant content. Goldhaber in Léveillé Gauvin (2018) mentions this phenomenon and relates it to economics of attention. Goldhaber shows that in cyberspace, a limited resource fought for is users' attention (Léveillé Gauvin 2018). One way to obtain users' attention in digital music streaming application is by showing differences through recommendations and playlists (Morris 2015).

\subsection{Streaming}

The practice of streaming has existed since the 1990s. Herbert et al. (2018) explain that streaming at that time was conceived as a process of sending media through the Internet without the need to download or store content in computer storage, with content presented in real time. Nowadays, streaming is differently conceptualized since it relates strictly to on-demand services and mainstream content. As one of its characteristics, streaming provides on-demand content. Meanwhile, Hesmondhalg and Meier (2017) in Herbert et al. (2018) mention that streaming services have two characteristics, a subscription mechanism and content transmitted via an Internet protocol.
Streaming practices offer different perspectives in digital content consumption, in this case, music content. In recording's early days, music was meant to be bought, and its physical artifacts were owned as records, vinyl albums, cassettes, or CDs. Now, however, buying music is being replaced by subscribing to access to music streaming providers (Mulligan 2018). Herbert et al. (2018) add to Mulligan's view that digital music streaming also involves a subscription mechanism. By subscribing, users gain access to service providers' catalog for a certain period, usually months or years.

\subsection{Wesley-Maclean Communication Model and Gatekeeping}

Developed from Wesley and Maclean's (Ruben \& Stewart 2006) communication model to describe the outwork of a newspaper editorial board, the gatekeeping model can also explain other communication phenomena. In a communication process, certain units run information selection and filtering functions to keep information relevant, according to the gatekeeper's goals.

Wesley and Maclean's (Ruben \& Stewart 2006) communication model is relevant to explaining playlists in digital music streaming applications. A playlist is a navigational tool for finding desired audio content. A playlist also filters or narrows available content. Thus, users can choose music effectively because filtered contents represent a certain context, in line with Bhaskar's (2006) curation process.

\subsection{Playlist}

The term "playlist" initially related to radio to describe an organized list with a number of songs selected for airplay (Straubhaar, LaRose, \& Davenport 2012). Another definition for playlist in the digital music platform is "song aggregation model" (Fuller 2017 in Meier \& Manzerolle 2018), meaning a digital music platform that selects varied content and uses technology for playbacks and presentations (Morris 2015b in Meier \& Manzerolle 2018). Fuller (in Meier \& Manzerolle 2018) also proposed that the playlist competes with the album as an aggregate model of songs.

As a basic function, a playlist is a navigational tool to organize a wide range and a large number of musical pieces (Herbert et al. 2018). Hagen (2015 in Herbert et al. 2018) states that a proper navigational technique is required for using musical content according to the situation. In digital music streaming applications, playlists can be made by the streaming service provider company (top-down) and its users (bottom-up) (Herbert et al. 2018). Audio streaming service providers see a playlist as a strategy to differentiate their services from those of their competitors (Prey 2018). A playlist also has commercial value because it can generate advertisement income. Therefore, the playlist must target users with the right profile (Prey 2018). A playlist also offers users freedom to list their desired songs, and users can share 
their playlists with others (Herbert et al. 2018), a characteristic differentiating playlists from music albums.

Despite use of algorithms for making playlists, the human touch guarantees maximum experience and ensures context relevance. For example, Spotify hires employees to create and perfect playlists (Buzzfeed News 2017; Ugwu 2016). At Spotify Indonesia, a position has been opened for a senior editor, responsible for curating playlists (Spotify n.d.).

\subsection{Curation}

Curation is an act of selecting or organizing things so that they gain value (Bhaskar 2016), especially organization of large numbers of things. Curation acts as a point of connection, mediator, membrane, or filter that balances consumers' needs and demands when facing abundance (Bhaskar 2016). Therefore, curation offers crucial value to those who live with abundance. A person's ability to curate information, lifestyle, or fashion becomes the crucial value in producing certain content and how cultural content is accepted (Morris, 2015).

Two main principles in curation are selection and arrangement (Bhaskar 2016). Selection means choosing certain knowledge about something to obtain the most significant output. Arrangement relates to changing how things are connected, that is, connections between interpersonal relations, images, words, ideas, history, and so on. During the curation process, a certain arrangement builds connection between things. A curator should be able to organize, differentiate, and build new patterns from existing ones.

In contrast, an algorithm curates music based on statistical data, such as song duration, genre, how many times users skip it, users' locations when accessing the song, time and season, and lists of songs to which a user has previously listened (Eriksson, Fleischer, Johansson, Snickars, \& Vonderau 2019). Meanwhile, a human curator works qualitatively, basing evaluations on how relevant listening to music is compared to how representative the songs in a certain playlist are. Therefore, human curators become a crucial aspect in perfecting playlists for recommendation to users. Human curators also show that curation by algorithm cannot replace the human touch.

\section{RESEARCH METHOD}

This article uses the literature review method as its research basis. Literature review attempts to combine the whole of knowledge on a topic (Neumann 2014) and to create a framework based on previous studies. From the literature review, researchers can identify which part of a topic has room for improvement. A literature review also aims to connect a current study with the phenomenon's big picture and to open new opportunities for future studies (Creswell 2014). Using literature review, this article aims to enrich the idea that curation in digital music streaming application is best achieved by humans and algorithms acting together, connecting expert human curators' capabilities with those of the algorithmin in a streaming platform.

The concepts discussed in detail in this article have already been mentioned: curation, playlist, gatekeeping, and digital music streaming, obtained from scientific journals and books. This article first pinpoints issues of digital music consumption according to Herbert et al. (2018) and to Lobato (2018), who mentions curated catalogs as characteristic of streaming services. Arditi (2017) sees access to a streaming source as an important digital streaming service. The next focus revisits work of Hesmondhalg and Meier (2017) and Herbert et al. (2018). The concepts of streaming and curated catalogs are then synthesized with Wesley and Maclean's (Ruben \& Stewart 2006) gatekeeping concept, which mentions information selection and filtering (Ruben \& Steward 2006). The playlist concept emerges from the works of Straubhaar, LaRose, and Davenport (2012), and Fuller (2017 in Meier \& Manzerolle 2018). All emergent concepts are then related to the concept of curation (Bhaskar 2016), that is, organizing things in large numbers and managing abundance.

Journals referenced here are no older than 5 years. To show that curation activity in digital music streaming also involves humans, the article refers to several secondary data sources, such as online news sites and YouTube pages with videos on Spotify editors. Several online news articles specifically report on Spotify's development in Indonesia.

Several studies have addressed the role of curation in recommending content. Most stress the curation process's use of algorithms, but few studies stress the human touch. Showing a curation process in digital video on the Netflix streaming platform, one study concerns changes in consumption patterns from scheduled consumption to consumption based on curated contents' availability (Lobato 2018). Besides Lobato's study (2018), several journals specifically discuss digital music streaming, using Spotify as an observation platform. Other studies see content curation as force that pushed streamed music consumption after digital music became trendy, that is, satisfying users' needs to obtain desired content after gaining access to certain music catalogs (Léveillé Gauvin 2018). Finally, other studies see Spotify playlists resulting from a curation process that also has other social dimensions and allows those dimensions to enter online space (Herbert et al. 2018).

\section{RESULTS AND DISCUSSION}

The literature review revealed that human curators play an important role in creating playlists for digital music streaming applications. Best practice is shown by combining the human with the algorithm curation process (Eriksson et al. 2019). This combination aims to maintain playlist quality and, thus, the quality of user experience. Besides, curated music formed into playlists can also be seen as part of the industry in digital audio streaming, opening job opportunities. 


\subsection{Experience of Listening to Streamed Music}

Today's digital music streaming platforms can have millions of songs in their catalogs, ready for users to access. Users no longer have to own the music file because the music they want is relatively easy to access from the Internet. Highlighting this phenomenon, Hamilton (2019) notes that streaming services offer users a music catalog that seems to be their own. The ready-to-access music catalog is currently a crucial factor forming the experience of listening to music through digital music streaming applications.

Torres (2018) also highlights the experience formed from changes in the music consumption pattern to streamed music. Applications used to listen to streamed music effect a different listening experience than other media because the application uses distinctive features and functions to help users navigate access to music content. In the platform, interactions occur between an algorithm and a recommendation system (Torres 2018). This interaction affects how users select songs for listening.

Streamed music's main characteristic is its consumption pattern or how users treat songs: A song used to be an owned content, but now, listeners access songs without needing the physical artifact (cassettes, CDs) or even digital files. Today, consuming music means accessing music content from a catalog that provides such content (Mulligan 2018). Mulligan (2018) sees this method of accessing music as beneficial to users. They no longer need to buy music stored in limited capacity artifacts or find space to store those artifacts. Audio streaming technology has allowed users to access catalogs of abundant songs when they subscribe to an available streamed music provider.

Herbert et al. (2018) see the strength of digital music streaming application as its "anytime access." To listen to a song, users simply use an electronic device or a computer connected to the Internet to access the online music catalog to which they are subscribed. This activity enhances listening experience when supported by other technologies, such as stereo headphones or high-fidelity speakers. In short, music is near and available anytime (Herbert et al. 2018).

\subsection{Content Curation, Playlists, and Content Recommendations}

Selecting music content based on a certain context underlies the concept of curation, resulting in playlists relevant to and recommended for particular contexts. Therefore, the curation process is actually crucial in selecting and organizing music content. After the selection process, content in a playlist has gained added value from the curation process (Bhaskar 2016).

Both users and service providers count on playlists to maximize use of digital streaming music applications, so, at this point, curation activity takes place. When facing abundant music content, users must decide what music they want to hear and find a way to filter music content (Bhaskar 2016). Certainly, music content curation differentiates one streaming service from others. Indeed, music services providers compete for better curation than their competitors in hopes of causing their applications to gain subscribers (Bhaskar 2016).

With many subscribers who listen regularly, one successful result of music curation in Spotify is Discover Weekly, a particular playlist that consists of specific music recommendations, personalized for each user. Discover Weekly's strong point is its ability to update its content each week. Updated on Mondays, Discover Weekly gives users the pleasure of discovering new music, performers, and songs. Eriksson et al. (2019) note that Discover Weekly has opened the opportunity for Spotify to add more types of playlist recommendations, for instance, Fresh Finds and Release Radar.

Pasnick (2015 in Hamilton 2019) mentioned Discover Weekly's complex mechanism for creating playlists worthy of recommendation. However, the application depends on data availability as the main source of information. An algorithm mixes consumer activity data and metadata about content. This combination is then used to create playlists that effectively recommend music to users (Zhu \& Chen 2018 in Eriksson et al. 2019). But Ananny (2016 in Eriksson et al. 2019) emphasizes that the algorithm curation process is very complex and carefully kept a company secret (Eriksson et al. 2019).

Discussing an algorithm's ability to create recommendations leads to a question. If the algorithm can do the job, why do streaming music service providers still need human editors? Even though an algorithm can sort music for listeners, in reality, the algorithm system is not autonomous; It cannot run independently. The algorithm and recommendation system actually results from interaction between humans and codes (Morris 2015). These interactions in the streaming platform are meant to give meaning to content. Indeed, an algorithm can read user data in the form of statistics, for example, how long a user listens to music each day, but this happens only because the algorithm is prepared beforehand. The possibility of misinterpreting statistical data when creating recommendations always exists, and such misinterpretation is likely to disturb users' listening experience. 


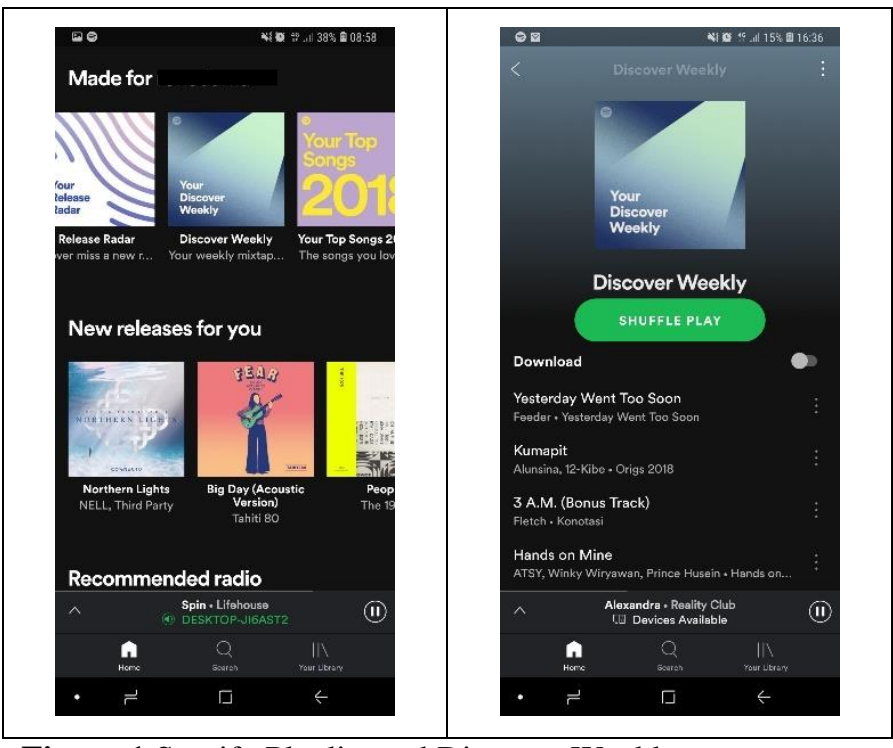

Figure 1 Spotify Playlist and Discover Weekly.

Bhaskar (2016) responds that human curators and algorithms work collaboratively in the Curated Model of Selection. This model shows that in the curation process, there lies a further process of making choices. Choices emerging from human and machine interaction become the best of both worlds. The Curated Model of Selection shows that choices emerge because of too much information, and curation filters this information to ease its organization. Therefore, the curation strengthens selection processes by applying both algorithmic and human capabilities to create effective recommendations.

Spotify's development of algorithm and human curation suitably exemplifies the curation relationship between human and machine. At first, Spotify engaged in providing music based on algorithms as its main service. However, users perceived Spotify as relying too heavily on algorithms and lacking the human touch. In fact, an algorithm's effectiveness depends on the people who design it (Morris 2015). In other words, an algorithm can provide results only if a user has been interacting with the application and has data recorded in the system; thus, the result is frequent recommendations of songs with which a user is already familiar. An algorithm alone does not work well if a user wants to find new music (Eriksson, Fleischer, Johansson, Snickars, \& Vonderau 2019).

Therefore, Spotify developed its service to accommodate human curation and playlist creation. By 2013, Spotify had hired curators to create playlists that resonated with local contexts in each country. Their job was to identify and execute playlists based on a certain mood or moment, different genres, and the local music scene in a region (Eriksson et al. 2019). Suffice it to say that the curating process in a streaming music service can be done in two ways: by curators or by algorithm. Spotify uses both methods, and the combination becomes best practice for digital music streaming applications.

Human curators exist in the position of "editor" in Spotify's organizational structure (Buzzfeed News 2017). In
2014, Spotify's curator team of 50 created more than 4,500 playlists in two years [this previous sentence needs revising because 2014 is not two years - the wording makes the sentence seem contradictory] (Ugwu 2016). As the streaming application trend grows, music streaming service providers offer more opportunity for music curators to contribute from within their platforms and create playlists.

The need for professional music content curators creates job opportunities. Spotify Indonesia has opened the position of Senior Editor, Shows and Editorial (Spotify n.d.). The position's job description includes the following eight tasks: (1) identifying and perfecting content hypotheses; (2) functioning as senior editor for the cultural division of the Indonesian music market; (3) presenting and following-up strategies and creative projects in Indonesia; (4) organizing and representing the Shows and Editorial Division for the Indonesian market; (5) curating most playlists and brand playlists and evaluating analytic data and work indicators; (6) working with the head of Shows and Editorial Indonesia and global; and (7) working with the global editorial team to achieve objectives.

This senior editor job description clearly states, in number 5 above, the curation function by human curators. Spotify also has strict requirements for the position: (1) more than 5 years' experience in the music industry; (2) deep knowledge of music and culture; (3) Bahasa Indonesia comprehension; (4) a good reputation in the music industry; (5) attention to detail; and (6) a passion for music (Spotify n.d.).

Creating a Spotify playlist is a form of service and has become a profession in the industry. Besides in-house work, Spotify also uses third-party services to curate music content, for instance, Filtr, Topsify, or Disgster (Eriksson et al. 2019). To perform in-house curation, Spotify adopts a curation mechanism from Tunigo, which Spotify acquired in May 2013. Tunigo applied a curation process based on certain activities, for example, playlists that represent a particular activity or mood--running, studying, or representing songs played in cafes (Eriksson et al. 2019).

Playlists offer not only a content navigation tool for users but can have added value. For example, Swedish radio broadcasts often talk about popular new or trending playlists. In Sweden, people commonly recommending playlists to one another. Playlists have also been attached to political values; the Swedish government is proud of Spotify. Former Swedish Prime Minister Carl Bildt used to give Spotify Premium accounts to guests as souvenirs, and they have been inserted into Swedish traditional music (Eriksson et al. 2019).

Besides being used in state programs, Spotify playlists can also be used to run protests. In Sweden also, the Twitter campaign \#backaspotify demonstrated that this digital music streaming application is expanding beyond being simply a provider of streamed music (Eriksson et al. 2019). 


\subsection{Content Curation and Gatekeeping}

In digital audio streaming applications, content curation can be seen as a gatekeeping process, that is, filtering information to make it more readily available to audiences (Ruben \& Stewart 2006). In Spotify, editors working as curators and curation algorithms run the gatekeeping function. Meanwhile, abundant music content must be filtered. When the gatekeeping process takes place, both curators and algorithms conduct the curation process by selecting and arranging plentiful, varied music content. Songs are filtered so that only those matching the curator's criteria are selected. The end result of the curation process is also the end result of the gatekeeping process, and the product is a playlist.

\section{CONCLUSION}

Streaming technology has brought significant changes to music lovers' ways of consuming music. In digital music streaming applications, users face millions of music selections. However, all these songs become irrelevant if users cannot choose which song to listen to because of too many choices and information overload (Jansson \& Hracs 2018). Therefore, users need a form of curation to help them navigate the digital music streaming application. Results of curation processes emerge as playlists, each with a particular context and relevant information, to help users gain the maximum music listening experience.

Additionally, media consumption patterns have changed because of streaming technology. Previously, music consumption was oriented toward ownership of a content asset. To consume a particular media content, an audience had to buy a cassette or a $\mathrm{CD}$, a physical artifact. When streaming technology entered the limelight, owning cassettes or CDs became irrelevant, and this was a major shift in media consumption, from record ownership to access ownership (Mulligan 2018). In the era of streaming, the more important question is selections' availability from a catalog. Does the streaming service's catalog contain the users' desired, varied, and abundant content?

Certainly curation capability is an important commodity in the era of information overload. The strong point of streaming platforms lies in whether content curation can help users navigate better (Herbert, Lotz, \& Marshall 2018). This becomes relevant since the quality of curation in playlists is the key factor that distinguishes a streaming platform from its competitors. Therefore, curation is at once a differentiating factor and a contributor of economic value (Jansson \& Hracs 2018).

This article emphasizes two elementary principles of curation--selection and arrangement (Bhaskar 2016). The curation discipline becomes relevant in consumption of all sorts of media, that is, curation is not limited only to digital audio streaming platforms. Exploration of curation and its principles shows that curation itself is multidisciplinary knowledge with principles that can be applied to wide variety of media.
In a digital music streaming application, music content curation is relevant to Wesley and Maclean's (Ruben \& Stewart 2006) communication model, which mentions the role of gatekeeper. In music streaming, the assigned gatekeepers are curators and curation algorithms that select and arrange content. They filter irrelevant content out of and relevant content into playlists, thus affirming curators' function as a gatekeeper.

In curating digital streaming content, the synergy between human curators and algorithms is a form of best practice in the streaming industry, that is, giving the best recommendations to users of a particular streaming application.

Finally, playlists not only function as a navigation tool for digital music streaming but can be attached to a political value. Moreover, a Spotify playlist can represent a collective aspiration, that is, songs with the spirit of activism can be curated into a particular playlist, thus acting as more than just a navigational tool.

\section{REFERENCES}

[1] Arditi, D. (2017) 'Music everywhere: Setting a digital music trap'. Critical Sociology, 089692051772919. https://doi.org/10.1177/0896920517729192

[2] Beer, D., and Burrows, R. (2013) 'Popular culture, digital archives and the new social life of data. Theory, culture \& society, 30(4), 47-71. https://doi.org/10.1177/0263276413476542

[3] Bhaskar, M. (2016) Curation the Power of Selection in a World of Excess. London: Piatkus.

[4] Bohang, F. K. (2016, September 6). Orang Indonesia Dengar Lagu di Spotify 90 Menit Sehari.

[5] Buzzfeed News. (2017) 'I Create Spotify Playlists for a Living', $\quad$ Available at: https://www.youtube.com/watch?v=Ji_WfHxatoQ (Accessed 26 March 2019)

[6] Creswell, J. W. (2014) 'Research Design Qualitative, Quantitative, and Mixed Methods Approaches' (Fourth Edi). California: Sage Publications, Inc.

[7] Eriksson, M., Fleischer, R., Johansson, A., Snickars, P., and Vonderau, P. (2019) Spotify Teardown. London: MIT Press.

[8] Hall, P. (2019) 'The Best Music Streaming Services'., Available at: https://www.digitaltrends.com/music/best-musicstreaming-services/ (Accessed 25 March 2019)

[9] Hamilton, C. (2019) 'Popular music, digital technologies and data analysis: New methods and questions'. Convergence: The International Journal of Research into New Media Technologies, 135485651983112. https://doi.org/10.1177/1354856519831127

[10] Herbert, D., Lotz, A. D., and Marshall, L. (2018) 
'Approaching media industries comparatively: A case study of streaming'. International Journal of Cultural Studies, $1-18$. https://doi.org/10.1177/1367877918813245

[11] Indihome. (n.d.). Indihome Musik | About Indihome. Accessed 25 March 2019, from http://www.melon.co.id/id/indihome/about

[12] Jansson, J., and Hracs, B. J. (2018) 'Conceptualizing curation in the age of abundance: The case of recorded music'. Environment and Planning A, 50(8), 16021625. https://doi.org/10.1177/0308518X18777497

[13] Kemp, S. 2019. 'Digital 2019 in Indonesia'.

[14] Léveillé Gauvin, H. (2018) 'Drawing listener attention in popular music: Testing five musical features arising from the theory of attention economy'. Musicae Scientiae, 22(3), 291-304. https://doi.org/10.1177/1029864917698010

[15] Lobato, R. (2018) 'Rethinking International TV Flows Research in the Age of Netflix'. Television and New Media, 19(3), 241-256. https://doi.org/10.1177/1527476417708245

[16] Meier, L. M., and Manzerolle, V. R. (2018) 'Rising tides? Data capture, platform accumulation, and new monopolies in the digital music economy'. New Media and Society. https://doi.org/10.1177/1461444818800998

[17] Morris, J. W. (2015) 'Curation by code: Infomediaries and the data mining of taste'. European Journal of Cultural Studies, 18(4-5), 446-463. https://doi.org/10.1177/1367549415577387

[18] Mulligan, M. (2018) 'From Ownership to Access'. Available at: http://www.midiaresearch.com/blog/from-ownershipto-access/ (Accessed March 26, 2019)

[19] Neumann, W. L. (2014) Social Research Methods: Quantitative and Qualitative Approaches. Pflege aktuell (Seventh Ed, Vol. 57). Essex: Pearson Education Limited. https://doi.org/10.2307/3211488

[20] Prey, R. (2018) 'Nothing personal: algorithmic individuation on music streaming platforms'. Media, Culture and Society, 40(7), 1086-1100. https://doi.org/10.1177/0163443717745147

[21] Ruben, B. D., and Stewart, L. P. (2006) Communication and Human Behavior (Fifth Edit). Boston: Pearson Education.

[22] Spotify. (n.d.). Senior Editor, Shows and EditorialIndonesia. Available at: https://www.spotifyjobs.com/job/senior-editor-showsand-editorial-indonesia-o5wl7fwd/ (Accessed 26 March 2019)

[23] Straubhaar, J., LaRose, R., and Davenport, L. (2012)
'Media Now: Understanding Media, Culture, and Technology' (2011)-1. Michael Rosenberg.

[24] Torres, V. Á. (2018) 'Book Review: Streaming music: Practices, media, cultures'. New Media \& Society, 146144481882007. https://doi.org/10.1177/1461444818820074

[25] Ugwu, R. (2016) 'Inside The Playlist Factory'. Available at: https://www.buzzfeed.com/reggieugwu/the-unsungheroes-of-the-music-streamingboom?utm_term=.diVGMx1Xb\#.oo0P0jzyZ (Accessed 26 March 2019)

[26] Vonderau, P. (2019) 'The Spotify Effect: Digital Distribution and Financial Growth'. Television and New Media, 20(1), 3-19. https://doi.org/10.1177/1527476417741200

[27] Werner, A., \& Johansson, S. (2016) 'Experts, dads and technology Gendered talk about online music'. International Journal of Cultural Studies, 19(2), 177192. https://doi.org/10.1177/1367877914555463 